\title{
Brownian Motion, Reflection Groups and Tanaka Formula
}

\author{
NiZAR Demni (*) - Dominique LéPINGLe (**)
}

ABSTRACT - In the setting of finite reflection groups, we prove that the projection of a Brownian motion onto a closed Weyl chamber is another Brownian motion normally reflected on the walls of the chamber. Our proof is probabilistic and the decomposition we obtain may be seen as a multidimensional extension of Tanaka's formula for linear Brownian motion. The paper is closed with a description of the boundary process through the local times of the distances from the initial process to the facets.

\section{Introduction}

The study of stochastic processes in relation to root systems has known a considerable growth during the last decade (see [4], [5] and references therein). In this paper, a reflection group $W$ associated with a root system $R$ is acting on a finite-dimensional Euclidean space $V$, and we project a $V$-valued Brownian motion onto the positive closed Weyl chamber $\bar{C}$. In fact, each $x \in V$ is conjugated to a unique $y \in \bar{C}$ so that the projection $\pi$ maps vectors in $V$ into their orbits under the action of $W$, namely $\pi: V \rightarrow V / W$. Using the terminology of Dunkl processes, $\pi(X)$ is often called the $W$-invariant or radial part of the initial Brownian motion $X$ ([4], [5]), yet should not be confused with the Brownian motion in a Weyl chamber considered in [1] which behaves as a Brownian motion conditioned to stay in the open chamber $C$. The main result of this paper is the explicit semimartingale decomposition of the projected process $\pi(X)$. Actually, the latter process is shown to behave as a $V$-valued Brownian motion in the interior of $\bar{C}$ and to reflect normally at the

(*) Indirizzo dell'A.: Université de Rennes I, IRMAR, F-35042 Rennes

E-mail: nizar.demni@univ-rennes1.fr

(**) Indirizzo dell'A.: Université d'Orléans, MAPMO-FDP, F-45067 Orléans

E-mail: dominique.lepingle@univ-orleans.fr 
boundary $\partial C$. The proof uses two ingredients of algebraic and probabilistic nature respectively : the expression of $\pi$ as the composition of a finite number of one dimensional projections and Tanaka formula for the absolute value of the linear Brownian motion. Another combination of tools from both reflection groups theory and probability theory leads to a precise description of the boundary process by means of the local time of the distance from $X$ to the facets.

The paper is organized as follows. Section 2 is devoted to basic facts on the theory of reflection groups and to a thorough study of reflections acting on facets of a root system. An illustration of this study is given in Section 3 where we investigate the evolution of a simple random walk on a triangular lattice in the plane with the dihedral group $\mathcal{D}_{3}$ operating on the lattice. We prove the main result in Section 4. Finally, Section 5 is devoted to the description of the boundary process. Note that the problem has previously been tackled in [3] and taken up again in [5], however the proof of Theorem 7.1 displayed in [5] p. 216 and quoted from [3] is not correct. Moreover, our approach is different and more general since we do not assume $\bar{C}$ to be a simplex, an assumption that ceases to hold for some root systems.

\section{Root systems and reflected facets}

We give a short introduction to the theory of reflection groups and refer to [6] for more details. Let $V$ be an Euclidean space with dimension $N$. For $\alpha \in V$ a unit vector we denote by $s_{\alpha}$ the orthogonal reflection with respect to the hyperplane $H_{\alpha}:=\{x \in V: \alpha . x=0\}$ :

$$
s_{\alpha}(x):=x-2(\alpha . x) \alpha .
$$

A finite subset $R$ of unit vectors in $V$ is called a reduced root system if for all $\alpha \in R$

$$
\begin{aligned}
& R \cap \mathbb{R} \alpha=\{\alpha,-\alpha\} ; \\
& s_{\alpha}(R)=R .
\end{aligned}
$$

The group $W \subset O(N)$ which is generated by the reflections $\left\{s_{\alpha}, \alpha \in R\right\}$ is called the reflection group associated with $R$. Each hyperplane $H_{\beta}:=\{x \in V: \beta . x=0\}$ with $\beta \in V \backslash \cup_{\alpha \in R} H_{\alpha}$ separates the root system $R$ into $R_{+}$and $R_{-}$. Such a set $R_{+}$is called a positive subsystem and defines the positive Weyl chamber $C$ by

$$
C:=\left\{x \in V: \alpha . x>0 \quad \forall \alpha \in R_{+}\right\} .
$$


A subset $S$ of $R_{+}$is called simple if $S$ is a vector basis for $\operatorname{span}(R)$ and if each positive root is a linear combination of elements of $S$ with coefficients being all nonnegative. The elements of $S$ are called simple roots. Such a subset exists and is unique once $R_{+}$is fixed. Moreover (Theorem 1.5 in [6]), $W$ is generated by the reflections $\left\{s_{\alpha}, \alpha \in S\right\}$. The positive Weyl chamber may also be written

$$
C=\{x \in V: \alpha . x>0 \quad \forall \alpha \in S\} .
$$

Its closure is called the closed Weyl chamber

$$
\bar{C}=\{x \in V: \alpha . x \geq 0 \forall \alpha \in S\} .
$$

With any partition $R_{+}=I_{+} \cup I_{0} \cup I_{-}$we associate the facet

$$
F=\left\{x \in V: \alpha . x>0 \quad \forall \alpha \in I_{+}, \beta . x=0 \quad \forall \beta \in I_{0}, \gamma \cdot x<0 \quad \forall \gamma \in I_{-}\right\} .
$$

We note $\left(I_{+}(F), I_{0}(F), I_{-}(F)\right)$ the partition corresponding to the facet $F$. We call $\mathcal{F}$ the set of nonempty facets with $\operatorname{Card}\left\{I_{0}\right\}=1$. For a facet $F \in \mathcal{F}$ with $I_{0}(F)=\{\beta\}, F \subset H_{\beta}$ and $H_{\beta}$ is called the support of $F$. The following result is an easy consequence of the isometry property of $s_{\alpha}$ and of the fact that $s_{\alpha}\left(R_{+} \backslash\{\alpha\}\right)=R_{+} \backslash\{\alpha\}$ (Proposition 1.4 in [6]).

Lemma 1. For $F \in \mathcal{F}$ and $\alpha \in S, s_{\alpha}(F) \in \mathcal{F}$ and

$$
\begin{aligned}
I_{0}\left(s_{\alpha}(F)\right) & =\{\alpha\} & \text { if } & I_{0}(F)=\{\alpha\} \\
& =s_{\alpha}\left(I_{0}(F)\right) & \text { if } & I_{0}(F) \neq\{\alpha\} .
\end{aligned}
$$

For $F \in \mathcal{F}$ and $w \in W, w(F) \in \mathcal{F}$ and

$$
\begin{aligned}
I_{0}(w(F)) & =w\left(I_{0}(F)\right) \quad \text { if } \quad w\left(I_{0}(F)\right) \in R_{+} \\
& =-w\left(I_{0}(F)\right) \quad \text { if } \quad w\left(I_{0}(F)\right) \in R_{-} .
\end{aligned}
$$

For any $\alpha \in S$ we write $F_{\alpha}$ for the facet $F$ associated with $I_{0}=\{\alpha\}, I_{-}=\emptyset$ :

$$
F_{\alpha}=\{x \in V: \alpha . x=0, \beta . x>0 \quad \forall \beta \in S \backslash\{\alpha\}\} .
$$

It is a face ([2], p. 61) of the chamber $C$ with support the wall $H_{\alpha}$. For any simple root $\alpha \in S$ we introduce the mapping $r_{\alpha}$ defined on $V$ by

$$
\begin{array}{rll}
r_{\alpha}(x) & :=x & \text { if } \alpha . x \geq 0 \\
& :=s_{\alpha}(x) & \text { if } \quad \alpha . x \leq 0 .
\end{array}
$$


A concise formula is

$$
r_{\alpha}(x)=x+2(\alpha . x)^{-} \alpha .
$$

Let $w \in W$, and let $w=s_{\alpha_{l}} \cdots s_{\alpha_{1}}$ be a reduced decomposition of $w$ where $\alpha_{i}, i=1, \ldots, l$ are simple roots and $l=l(w)$ is the length of $w$. From Theorem 2.4 in [1] and a result of Matsumoto ([2], Ch.I V, No.1.5, Prop. 5) we know that the operator $r_{\alpha_{l}} \cdots r_{\alpha_{1}}$ depends only on $w$ and not on a particular reduced decomposition. We denote $r_{w}$ this operator. Of particular interest is the operator $r_{w_{0}}$ where $w_{0}$ denotes the (unique) longest element in $W: l\left(w_{0}\right)=\operatorname{Card}\left(R_{+}\right)$. The following lemma follows immediately from Corollary 2.9 in [1] and plays a key role in the proof of our main result.

LEMMA 2. If $w_{0}$ is the longest element in $W$, then $r_{w_{0}}$ takes values in the closed Weyl chamber $\bar{C}$ and for any $x \in V, \pi(x):=r_{w_{0}}(x)$ is the unique point of the orbit $W . x$ which lies in $\bar{C}$.

The following lemmas shed some light on the action of $r_{\alpha}$ and $\pi$.

LEMMA 3. Let $\alpha \in S$.

(1) $x \in \cup_{\beta \in R+} H_{\beta} \Longleftrightarrow r_{\alpha}(x) \in \cup_{\beta \in R+} H_{\beta}$.

(2) Let $F \in \mathcal{F}$ with support $H_{\beta}$.

(a) - If $\alpha \in I_{+}(F)$, then $r_{\alpha}(F)=F$.

- If $\{\alpha\}=I_{0}(F)$, then $r_{\alpha}(F)=F$ and $\alpha=\beta$.

- If $\alpha \in I_{-}(F)$, then $r_{\alpha}(F) \in \mathcal{F}$ and $r_{\alpha}(F) \subset H_{\gamma}$ with $\gamma=s_{\alpha}(\beta) \in R_{+}$.

(b) - If $\alpha \in I_{+}(F)$, then $r_{\alpha}^{-1}(F)=F \cup s_{\alpha}(F)$.

- If $\{\alpha\}=I_{0}(F)$, then $r_{\alpha}^{-1}(F)=F$ and $\alpha=\beta$.

- If $\alpha \in I_{-}(F)$, then $r_{\alpha}^{-1}(F)=\emptyset$.

Proof. (1) From the isometry property of $s_{\alpha} \in O(N)$ we derive that for $x \in V$ and $\beta \in R_{+}$

$$
\begin{aligned}
\beta . x & =s_{\alpha}(\beta) . s_{\alpha}(x) \\
s_{\alpha}(\beta) . x & =\beta . s_{\alpha}(x) .
\end{aligned}
$$

From Proposition 1.4 in [6] we know that $s_{\alpha}\left(R_{+} \backslash\{\alpha\}\right)=R_{+} \backslash\{\alpha\}$. Both implications $\Rightarrow$ and $\Leftarrow$ then follow.

(2) (a) The proofs of both the first and the second assertions are straightforward. Let $\alpha \in I_{-}(F), x \in F$ and $\delta \in R_{+}$. Then,

$$
\delta . x=s_{\alpha}(\delta) . s_{\alpha}(x)=s_{\alpha}(\delta) \cdot r_{\alpha}(x) .
$$


Therefore,

$$
\begin{aligned}
& \text { if } \delta=\alpha, \quad \alpha . r_{\alpha}(x)>0 \\
& \text { if } \delta=\beta, \quad s_{\alpha}(\beta) \cdot r_{\alpha}(x)=0 \\
& \text { if } \delta \in I_{+}(F), \quad s_{\alpha}(\delta) \cdot r_{\alpha}(x)>0 \\
& \text { if } \delta \in I_{-}(F) \backslash\{\alpha\}, \quad s_{\alpha}(\delta) \cdot r_{\alpha}(x)<0
\end{aligned}
$$

and using again Proposition 1.4 in [6] we get a new partition $\left(I_{+}=s_{\alpha}\left(I_{+}(F)\right) \cup\{\alpha\}, I_{0}=\left\{s_{\alpha}(\beta)\right\}, I_{-}=s_{\alpha}\left(I_{-}(F)\right) \backslash\{\alpha\}\right)$ corresponding to a new facet $r_{\alpha}(F)$.

(b) The proofs are straightforward or similar to the proofs in (a).

LeMma 4. (1) $x \in \cup_{\beta \in R+} H_{\beta} \Longleftrightarrow \pi(x) \in \partial C$.

(2) If $F \in \mathcal{F}$, there exists $\alpha \in S$ such that $\pi(F)=F_{\alpha}$.

(2) For $\alpha \in S, \pi^{-1}\left(F_{\alpha}\right)=\cup_{F \in \mathcal{F}_{\alpha}} F$ where we set

$$
\mathcal{F}_{\alpha}:=\left\{F \in \mathcal{F}: \pi(F)=F_{\alpha}\right\} .
$$

Proof. The first assertion is a consequence of (1) of Lemma 3 and of the property $\pi(V)=\bar{C}$. We also obtain from (2.a) of Lemma 3 that $\pi(F) \in \mathcal{F}$ and it follows there exists $\alpha \in S$ such that $\pi(F)=F_{\alpha}$. For $\alpha \in S$ we deduce from (2.b) of Lemma 3 that $\pi^{-1}\left(F_{\alpha}\right)$ is the union of facets from $\mathcal{F}$. Exactly all facets in $\mathcal{F}_{\alpha}$ are involved.

\section{Random walk on a triangular lattice}

Take $V$ to be the Euclidean plane $\mathbb{R}^{2}$. To each $(i, j) \in Z_{s}^{2}$ we associate a vertex in the plane with coordinates

$$
x_{(i, j)}=i+\frac{1}{2} j \quad y_{(i, j)}=\frac{\sqrt{3}}{2} j .
$$

The simple random walk $\left(Z_{n}\right)_{n \geq 0}$ on this lattice $T$ is a Markov chain with uniform transition probability $p((i, j),(k, l))=1 / 6$ on the six nearest neighbors $(k, l)$ of the vertex $(i, j)$. We now consider the dihedral group $\mathcal{D}_{3}$ consisting of six orthogonal transformations that preserve $\mathbb{T}$. We take $\alpha=(0,1)$ and $\beta=(\sqrt{3} / 2,-1 / 2)$ to be the simple roots, and $\gamma=(\sqrt{3} / 2,1 / 2)$ to be the third positive root. The discrete positive Weyl chamber $C_{d i s}$ is given by $\left\{(i, j) \in Z^{2}\right.$ : $i>0, j>0\}$. 


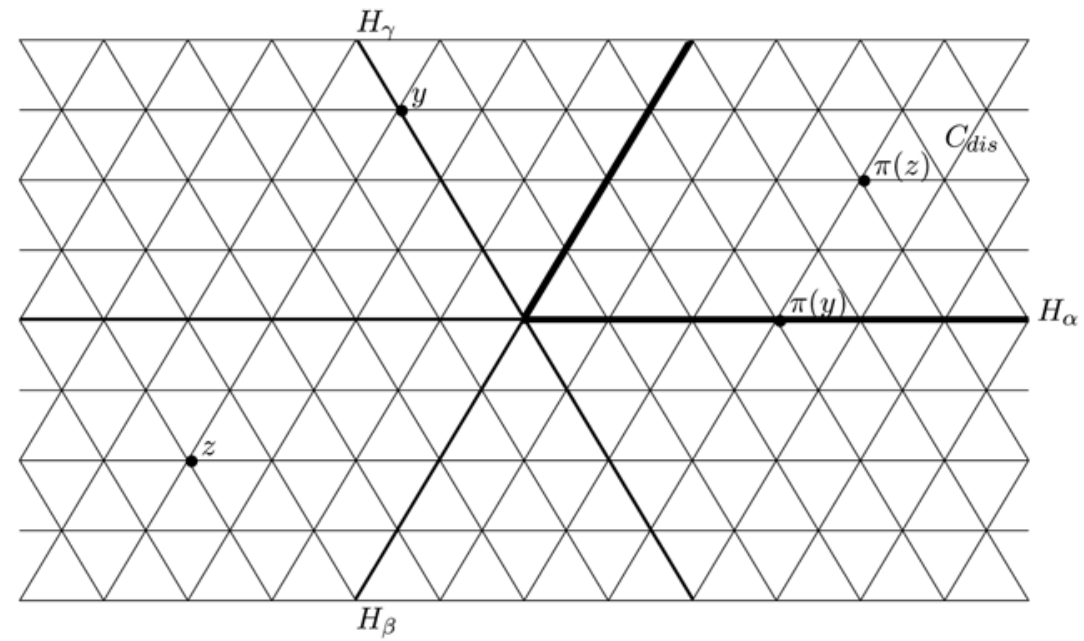

We note that $\pi=r_{\alpha} r_{\beta} r_{\alpha}=r_{\beta} r_{\alpha} r_{\beta}$. It is easily seen that $\left(\pi\left(Z_{n}\right)\right)_{n \geq 0}$ is a Markov chain on the set of vertices $\{(i, j) \in T: i \geq 0, j \geq 0\}$ with transition probability

$$
\begin{array}{ll}
q((i, j),(k, l))=p((i, j),(k, l)) & \text { if } i>0, j \\
q((i, 0),(i, 1))=q((i, 0),(i-1,1))=2 q((i, 0),(i \pm 1,0))=\frac{1}{3} & \text { if } i>0 \\
q((0, j),(1, j))=q((0, j),(1, j-1))=2 q((0, j),(0, j \pm 1))=\frac{1}{3} & \text { if } j>0 \\
q((0,0),(0,1))=q((0,0),(1,0))=\frac{1}{2} . &
\end{array}
$$

This is exactly what we should call the simple random walk normally reflected on the walls of the Weyl chamber $C_{d i s}$.

The examination of a simple random walk on a square or hexagonal lattice would lead us to the same conclusion.

\section{Brownian motion in a Weyl chamber}

We consider a probability space $(\Omega, \mathscr{A}, \mathbb{P})$ endowed with a right-continuous filtration $\left(\mathscr{C}_{t}\right)_{t \geq 0}$ and a $V$-valued Brownian motion $X$ with decomposition

$$
X_{t}=X_{0}+B_{t}
$$


We are interested in the continuous process $\pi(X)$. It can be seen as a $W$ invariant Dunkl process of zero multiplicity function. As such it is a Markov process with values in $\bar{C}$ and semi-group density ([5], p. 216):

$$
p_{t}(x, y)=\frac{1}{c_{0} t^{N / 2}} \exp \left\{-\frac{|x|^{2}+|y|^{2}}{2 t}\right\} \sum_{w \in W} \exp \left(\frac{1}{t} x . w(y)\right)
$$

where $c_{0}$ is a normalizing constant. We remark that for any fixed $y \in \bar{C}$ the function $p_{t}(x, y)$ is a solution to the heat equation with Neumann boundary conditions:

$$
\begin{aligned}
& \partial_{t} p_{t}=\frac{1}{2} \Delta p_{t} \quad \text { in } C \\
& \frac{\partial p_{t}}{\partial n}=0 \quad \text { on } \partial C
\end{aligned}
$$

where $\partial p_{t} / \partial n$ is any outward normal derivative of $p_{t}$. Thus there is no surprise when asserting that $\pi(X)$ should be a Brownian motion normally reflected on the boundary of $C$. The aim of this section is to obtain a decomposition of the continuous semimartingale $\pi(X)$. We first investigate the action of the operator $r_{\alpha}$ on continuous $V$-valued semimartingales. To proceed, recall that if $Z$ is a continuous real semimartingale with local time $L(Z)$ at 0 , then Tanaka formula shows that $Z^{-}$is still a semimartingale that decomposes as:

$$
Z_{t}^{-}=Z_{0}^{-}-\int_{0}^{t} \mathbf{1}_{\left\{Z_{s} \leq 0\right\}} d Z_{s}+\frac{1}{2} L_{t}(Z) .
$$

Lemma 5. Let $Y$ be a continuous $V$-valued semimartingale with Doob decomposition

$$
Y_{t}=Y_{0}+C_{t}+A_{t}
$$

where $C$ is a Brownian motion and $A$ is a continuous process of finite variation, both vanishing at time 0 . Then $Y^{\prime}=r_{\alpha}(Y)$ has a similar decomposition

$$
Y_{t}^{\prime}=Y_{0}^{\prime}+C_{t}^{\prime}+A_{t}^{\prime}
$$

where $Y_{0}^{\prime}=r_{\alpha}\left(Y_{0}\right), C^{\prime}$ is a Brownian motion given by

$$
C_{t}^{\prime}=\int_{0}^{t} O_{s}^{\prime} \cdot d C_{s}
$$


with $O^{\prime}$ a $O(N)$-valued process, and the finite variation process $A^{\prime}$ is given by

$$
A_{t}^{\prime}=A_{t}-2 \int_{0}^{t} \mathbf{1}_{\left\{\alpha . Y_{s} \leq 0\right\}} d\left(\alpha . A_{s}\right) \alpha+L_{t}(\alpha . Y) \alpha .
$$

Proof. We combine (3) with (4). Then

$$
\begin{aligned}
r_{\alpha}\left(Y_{t}\right) & =Y_{t}+2\left(\alpha . Y_{0}\right)^{-} \alpha-2 \int_{0}^{t} \mathbf{1}_{\left\{\alpha . Y_{s} \leq 0\right\}} d\left(\alpha . Y_{s}\right) \alpha+L_{t}(\alpha . Y) \alpha \\
& =r_{\alpha}\left(Y_{0}\right)+C_{t}-2 \int_{0}^{t} \mathbf{1}_{\left\{\alpha . Y_{s} \leq 0\right\}} d\left(\alpha . C_{s}\right) \alpha+A_{t}^{\prime} .
\end{aligned}
$$

The process $O^{\prime}$ defined by

$$
O_{s}^{\prime} . u=u-2 \mathbf{1}_{\left\{\alpha . Y_{s} \leq 0\right\}}(\alpha . u) \alpha
$$

for $u \in V$ satisfies

$$
\left(O_{s}^{\prime} \cdot u\right) \cdot\left(O_{s}^{\prime} \cdot v\right)=u \cdot v
$$

for any $u, v \in V$. It follows ([7], Ch. IV, Ex. 3.22) that

$$
C_{t}-2 \int_{0}^{t} \mathbf{1}_{\left\{\alpha . Y_{s} \leq 0\right\}} d\left(\alpha . C_{s}\right) \alpha=\int_{0}^{t} O_{s}^{\prime} . d C_{s}
$$

defines a $V$-valued Brownian motion.

We are now in a position to prove our main result. It states that a Brownian motion projected onto the closed Weyl chamber is another Brownian motion normally reflected on the faces of the chamber.

Theorem 6. Let $X_{t}=X_{0}+B_{t}$ be a V-valued Brownian motion. Then

$$
\pi\left(X_{t}\right)=\pi\left(X_{0}\right)+\int_{0}^{t} O_{s} . d B_{s}+\frac{1}{2} \sum_{\alpha \in S} L_{t}(\alpha . \pi(X)) \alpha
$$

where $O$ is a $O(N)$-valued process.

Proof. a) Let $\pi=r_{\alpha_{l}} \cdots r_{\alpha_{1}}$. We proceed by iteration, setting $X_{t}^{0}:=X_{t}, B_{t}^{0}:=B_{t}, A_{t}^{0}:=0$ and $X_{t}^{j}:=r_{\alpha_{j}}\left(X_{t}^{j-1}\right)$ for $j=1, \ldots, l$. If we de- 
compose

$$
X_{t}^{j}=X_{0}^{j}+B_{t}^{j}+A_{t}^{j}
$$

then Lemma 5 yields

$$
\begin{aligned}
& X_{0}^{j}=r_{\alpha_{j}}\left(X_{0}^{j-1}\right) \\
& B_{t}^{j}=\int_{0}^{t} O_{s}^{j} \cdot d B_{s}^{j-1} \\
& A_{t}^{j}=A_{t}^{j-1}-2 \int_{0}^{t} \mathbf{1}_{\left\{\alpha_{j} \cdot X_{s}^{j-1} \leq 0\right\}} d\left(\alpha_{j} \cdot A_{s}^{j-1}\right) \alpha_{j}+L_{t}\left(\alpha_{j} \cdot X^{j-1}\right) \alpha_{j},
\end{aligned}
$$

with $O^{j}$ a $O(N)$-valued process. Setting

$$
O:=O^{l} \cdots O^{1}
$$

we obtain the Brownian term in the decomposition of $\pi(X)=X^{l}$.

b) From the recurrence formula (5) we deduce that $d A_{t}^{l}$ is supported by $\cup_{j=1}^{l}\left\{X_{t}^{j-1} \in H_{\alpha_{j}}\right\}$ which is included in $\left\{X_{t} \in \cup_{\beta \in R_{+}} H_{\beta}\right\}$ by (1) of Lemma 3. Moreover, since one-points sets are polar sets for the planar Brownian motion, we remark that for any $t>0$, the Brownian motion $X$ does not meet facets with $\operatorname{Card}\left\{I_{0}\right\}>1$. Therefore

$$
\mathbf{1}_{\left\{X_{t} \in \cup_{\beta \in R_{+}} H_{\beta}\right\}}=\sum_{F \in \mathcal{F}} \mathbf{1}_{\left\{X_{t} \in F\right\}} \quad \text { a.s. }
$$

and we only need to study the sequence $\left(A^{j}\right)_{j=1, \ldots, l}$ on the sets $\left\{X_{t} \in F\right\}$ for any $F \in \mathcal{F}$. Set $F_{0}:=F$ and $F_{j}:=r_{\alpha_{j}}\left(F_{j-1}\right)$ for $j=1, \ldots, l$. Let $H_{\beta_{j}}$ be the support of the facet $F_{j}$. We will prove by induction that for any $j=0, \ldots, l$

$$
\mathbf{1}_{\left\{X_{t} \in F\right\}} d A_{t}^{j}=d L_{t}^{j} \beta_{j}
$$

where $L^{j}$ is an increasing process. This is true for $j=0$ with $L_{t}^{0}=0$. Assume this is true for $j-1$ and use (5). Then

$$
\begin{aligned}
& \mathbf{1}_{\left\{X_{t} \in F\right\}} d A_{t}^{j} \\
& =\mathbf{1}_{\left\{X_{t} \in F\right\}}\left[d L_{t}^{j-1} \beta_{j-1}-2 \mathbf{1}_{\left\{\alpha_{j} \cdot X_{s}^{j-1} \leq 0\right\}}\left(\alpha_{j} . \beta_{j-1}\right) d L_{t}^{j-1} \alpha_{j}+d L_{t}\left(\alpha_{j} . X^{j-1}\right) \alpha_{j}\right] .
\end{aligned}
$$

We apply Lemma 3. 
If $\alpha_{j} . x>0$ for $x \in F_{j-1}$, then $F_{j}=F_{j-1}, \beta_{j}=\beta_{j-1} \neq \alpha_{j}$ and

$$
\mathbf{1}_{\left\{X_{t} \in F\right\}} d A_{t}^{j}=d L_{t}^{j-1} \beta_{j-1}=d L_{t}^{j-1} \beta_{j} .
$$

If $\alpha_{j} . x=0$ for $x \in F_{j-1}$, then $F_{j}=F_{j-1}, \beta_{j}=\beta_{j-1}=\alpha_{j}$ and

$$
\mathbf{1}_{\left\{X_{t} \in F\right\}} d A_{t}^{j}=\left[-d L_{t}^{j-1}+\mathbf{1}_{\left\{X_{t} \in F\right\}} d L_{t}\left(\alpha_{j} \cdot X^{j-1}\right)\right] \beta_{j} .
$$

If $\alpha_{j} . x<0$ for $x \in F_{j-1}$, then $\alpha_{j} \neq \beta_{j-1}, \beta_{j}=s_{\alpha_{j}}\left(\beta_{j-1}\right)$ and

$$
\mathbf{1}_{\left\{X_{t} \in F\right\}} d A_{t}^{j}=d L_{t}^{j-1} \beta_{j} .
$$

In the first and third cases $L^{j}=L^{j-1}$ is increasing from the induction assumption. In the second case we use Tanaka formula again. As

$$
\alpha_{j} . X_{t}^{j}=\alpha_{j} . r_{\alpha_{j}}\left(X_{t}^{j-1}\right) \geq 0
$$

we get

$$
\begin{aligned}
\alpha_{j} \cdot X_{t}^{j} & =\left(\alpha_{j} \cdot X_{t}^{j}\right)^{+} \\
& =\left(\alpha_{j} \cdot X_{0}^{j}\right)^{+}+\int_{0}^{t} \mathbf{1}_{\left\{\alpha_{j} \cdot X_{s}^{j}>0\right\}} d\left(\alpha_{j} \cdot X_{s}^{j}\right)+\frac{1}{2} L_{t}\left(\alpha_{j} \cdot X^{j}\right)
\end{aligned}
$$

and therefore

$$
\begin{aligned}
d L_{t}^{j} & =\mathbf{1}_{\left\{X_{t} \in F\right\}} d\left(\alpha_{j} \cdot A_{t}^{j}\right) \\
& =\mathbf{1}_{\left\{X_{t} \in F\right\}}\left(\mathbf{1}_{\left\{\alpha_{j} \cdot X_{t}^{j}>0\right\}} d\left(\alpha_{j} \cdot A_{t}^{j}\right)+\frac{1}{2} d L_{t}\left(\alpha_{j} \cdot X^{j}\right)\right) \\
& =\frac{1}{2} \mathbf{1}_{\left\{X_{t} \in F\right\}} d L_{t}\left(\alpha_{j} \cdot X^{j}\right),
\end{aligned}
$$

which proves that $L^{j}$ is an increasing process.

c) We have obtained that for each $F \in \mathcal{F}$ there exists an increasing process $L^{l}$ such that

$$
\mathbf{1}_{\left\{X_{t} \in F\right\}} d A_{t}^{l}=d L_{t}^{l} \beta_{l} .
$$

From Lemma 4 we deduce that $F_{l}=\pi(F)=F_{\alpha}$ for some $\alpha \in S$, which entails $\beta_{l}=\alpha$. Take $L^{\alpha}$ to be the sum of the $L^{l}$ 's for all $F \in \mathcal{F}_{\alpha}$. Clearly $d L_{t}^{\alpha}$ is supported by

$$
\begin{aligned}
\cup_{F \in \mathcal{F}_{\alpha}}\left\{X_{t} \in F\right\} & =\left\{X_{t} \in \cup_{F \in \mathcal{F}_{\alpha}} F\right\} \\
& =\left\{\pi\left(X_{t}\right) \in F_{\alpha}\right\}
\end{aligned}
$$

where the last equality derives from (3) in Lemma 4. Summarizing 
we get

$$
\begin{aligned}
d A_{t}^{l} & =\mathbf{1}_{\left\{X_{t} \in \cup_{F \in \mathcal{F}}\right\}} d A_{t}^{l} \\
& =\sum_{\alpha \in S} \sum_{F \in \mathcal{F}_{\alpha}} \mathbf{1}_{\left\{X_{t} \in F\right\}} d A_{t}^{l} \\
& =\sum_{\alpha \in S} d L_{t}^{\alpha} \alpha .
\end{aligned}
$$

d) It remains to identify the boundary processes $L^{\alpha}$. We use the method in b) again. As

$$
\alpha . \pi\left(X_{t}\right) \geq 0
$$

we get

$$
\begin{aligned}
\alpha . \pi\left(X_{t}\right) & =\left(\alpha . \pi\left(X_{t}\right)\right)^{+} \\
& =\left(\alpha . \pi\left(X_{0}\right)\right)^{+}+\int_{0}^{t} \mathbf{1}_{\left\{\alpha . \pi\left(X_{s}\right)>0\right\}} d\left(\alpha . \pi\left(X_{s}\right)\right)+\frac{1}{2} L_{t}(\alpha . \pi(X)) .
\end{aligned}
$$

On the one hand,

$$
\begin{aligned}
\mathbf{1}_{\left\{\alpha . \pi\left(X_{t}\right)=0\right\}} d\left(\alpha . \pi\left(X_{t}\right)\right) & =\mathbf{1}_{\left\{\alpha \cdot \pi\left(X_{t}\right)=0\right\}}\left(\alpha \cdot\left(O_{s} \cdot d B_{s}\right)+\sum_{\beta \in S} d L_{t}^{\beta} \alpha . \beta\right) \\
& =d L_{t}^{\alpha}
\end{aligned}
$$

since the set $\left\{\alpha . \pi\left(X_{t}\right)=0\right\}$ has zero Lebesgue measure and $d L_{t}^{\beta}$ is supported by $\left\{\pi\left(X_{t}\right) \in F_{\beta}\right\}$ for $\beta \neq \alpha$. On the other hand,

$$
\mathbf{1}_{\left\{\alpha . \pi\left(X_{t}\right)=0\right\}} d\left(\alpha . \pi\left(X_{t}\right)\right)^{+}=\frac{1}{2} d L_{t}(\alpha . \pi(X))
$$

and we are done.

\section{Complements on the boundary process}

We have already seen in the previous section that on each face $F_{\alpha}$ of the Weyl chamber the boundary process is

$$
L_{t}^{\alpha}=\frac{1}{2} d L_{t}(\alpha . \pi(X))
$$

From Corollary VI.1.9 in [7] we know that this is a.s.

$$
\lim _{\varepsilon \downarrow 0} \frac{1}{2 \varepsilon} \int_{0}^{t} \mathbf{1}_{[0, \varepsilon)}\left(\alpha . \pi\left(X_{s}\right)\right) d s
$$


for every $t$. In the sequel, we seek for an expression of $L^{\alpha}$ involving the original process $X$ rather than the reflected one $\pi(X)$. For $x \in V$ and $A \subset V$ we shall use the standard notation

$$
d(x, A):=\inf \{|x-y|: y \in A\}
$$

and for $\alpha \in S$ define

$$
K_{\alpha}:=\pi^{-1}\left(F_{\alpha}\right)=\cup_{w \in W} w\left(F_{\alpha}\right) .
$$

Proposition 7. For any $x \in V$ and $\alpha \in S$,

$$
\alpha . \pi(x)=d\left(x, K_{\alpha}\right) .
$$

Proof. For any $\varepsilon>0$ there exists $y \in \pi^{-1}\left(F_{\alpha}\right)$ such that

$$
\begin{aligned}
\varepsilon+d\left(x, K_{\alpha}\right) & \geq|x-y| \\
& \geq|\pi(x)-\pi(y)| \\
& \geq \alpha \cdot(\pi(x)-\pi(y)) \\
& =\alpha \cdot \pi(x),
\end{aligned}
$$

where we have used the contraction property of $\pi$. In fact it is easy to check that each $r_{\beta}$ is contracting and so is $\pi$ by iteration, which proves that $d\left(x, K_{\alpha}\right) \geq \alpha . \pi(x)$. Conversely, let $x \in V$ and let $w \in W$ be such that $w(x)=\pi(x) \in \bar{C}$. Let us consider the facet $w^{-1}\left(F_{\alpha}\right)$. Using that $\alpha . \beta \leq 0$ for any $\alpha, \beta \in S$ with $\alpha \neq \beta$ ([6]) we check that

$$
x-(\alpha . w(x)) w^{-1}(\alpha) \in w^{-1}\left(F_{\alpha}\right) \subset K_{\alpha}
$$

and therefore

$$
d\left(x, K_{\alpha}\right) \leq \alpha . w(x)=\alpha . \pi(x) .
$$

We obtain a new expression for the boundary process:

$$
L_{t}^{\alpha}=\lim _{\varepsilon \downarrow 0} \frac{1}{2 \varepsilon} \int_{0}^{t} \mathbf{1}_{\left\{d\left(X_{s}, K_{\alpha}\right)<\varepsilon\right\}} d s .
$$

A little more can be said in a particular case.

Proposition 8. If $\alpha \in S$ is the only simple root in its orbit $R_{\alpha}:=W . \alpha$, then

$$
\overline{K_{\alpha}}=\cup_{\gamma \in R_{\alpha} \cap R_{+}} H_{\gamma}
$$


and

$$
L_{t}^{\alpha}=\sum_{\gamma \in R_{\alpha} \cap R_{+}} L_{t}(\gamma \cdot X)
$$

Proof. Let $w \in W$ and $F \in \mathcal{F}$ satisfy $F=w\left(F_{\alpha}\right)$. Let $G \in \mathcal{F}$ have same support $H_{\gamma}\left(\gamma \in R_{+}\right)$as $F$. There exist $\beta \in S$ and $w^{\prime} \in W$ such that $G=w^{\prime}\left(F_{\beta}\right)$. Then from Lemma 1

$$
\gamma= \pm w(\alpha)= \pm w^{\prime}(\beta)
$$

and $\alpha$ and $\beta$ are conjugate. It follows from the hypothesis that $\beta=\alpha$, and actually the whole hyperplane $H_{\gamma}$ belongs to $\overline{K_{\alpha}}$. Moreover $\overline{K_{\alpha}}$ is the union of all hyperplanes $H_{\gamma}$ with $\gamma$ a positive root in the orbit of $\alpha$. Now the difference

$$
\sum_{\gamma \in R_{\alpha} \cap R_{+}} \mathbf{1}_{\left\{\left|\gamma \cdot X_{s}\right|<\varepsilon\right\}}-\mathbf{1}_{\left\{\min _{\gamma \in R_{\alpha} \cap R_{+}}\left|\gamma \cdot X_{s}\right|<\varepsilon\right\}}
$$

is nonnegative and bounded above by a finite sum of terms of the form

$$
\mathbf{1}_{\left\{\left|\gamma_{1}, X_{s}\right|<\varepsilon,\left|\gamma_{2}, X_{s}\right|<\varepsilon\right\}}
$$

where $\gamma_{1}$ and $\gamma_{2}$ are independent unit vectors. Let $Y$ be the orthogonal projection of $X$ onto the two-dimensional space generated by $\gamma_{1}$ and $\gamma_{2}$. Then there exists $c>0$ such that

$$
\mathbf{1}_{\left\{\left|\gamma_{1}, X_{s}\right|<\varepsilon,\left|\gamma_{2}, X_{s}\right|<\varepsilon\right\}} \leq \mathbf{1}_{\left\{\left|Y_{s}\right|<c \varepsilon\right\}}
$$

and since the two-dimensional Bessel process $|Y|$ has local time zero at 0 it follows that a.s.

$$
\lim _{\varepsilon \downarrow 0} \frac{1}{2 \varepsilon} \int_{0}^{t} \mathbf{1}_{\left\{\left|\gamma_{1}, X_{s}\right|<\varepsilon,\left|\gamma_{2}, X_{s}\right|<\varepsilon\right\}} d s=0
$$

Thus

$$
L_{t}^{\alpha}=\lim _{\varepsilon \downarrow 0} \frac{1}{2 \varepsilon} \int_{0}^{t} \sum_{\gamma \in R_{\alpha} \cap R_{+}} \mathbf{1}_{\left\{\left|\gamma \cdot X_{s}\right|<\varepsilon\right\}} d s=\sum_{\gamma \in R_{\alpha} \cap R_{+}} L_{t}(\gamma \cdot X) .
$$

We now give two illustrative examples.

DIHEDRAL GROUP OF ORDER 8. This group consists of the orthogonal transformations which preserve a square centered at the origin in the plane $V=\mathbb{R}^{2}$. There are two simple roots $\alpha=\frac{1}{\sqrt{2}}\left(e_{1}-e_{2}\right), \beta=e_{2}$ which lie in two different orbits. The claim of the previous proposition for the simple 
root $\alpha$ is illustrated by the following picture:

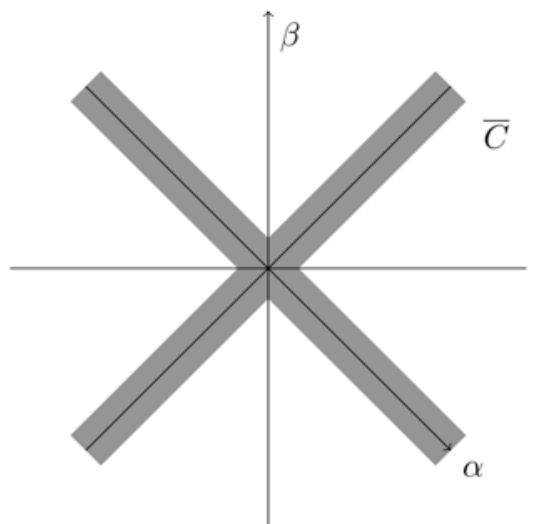

DIHEDRAL GROUP OF ORDER 6. We come back to the example considered in Section 3 and identify $\mathbb{R}^{2}$ with the complex plane $\mathrm{C}$. There are six twodimensional roots represented in complex notations as

$$
\left\{ \pm e^{-i \pi / 2} e^{i m \pi / 3}, m=1,2,3\right\}
$$

and there is only one orbit so that the hypothesis of the proposition breaks down. The reflection group $W=\mathcal{D}_{3}$ contains three rotations of angles $2 m \pi / 3, m \in\{1,2,3\}$ and three reflections $\mathbb{C} \ni z \mapsto \bar{z} e^{2 i m \pi / 3}$. When choosing $S=\{\alpha, \beta\}=\left\{e^{i \pi / 2}, e^{-i \pi / 6}\right\}, \bar{C}$ is a wedge of angle $\pi / 3$ in the positive quadrant of the plane and

$$
\left\{d\left(x, K_{\alpha}\right)<\varepsilon\right\}
$$

is the hachured part of the following picture:

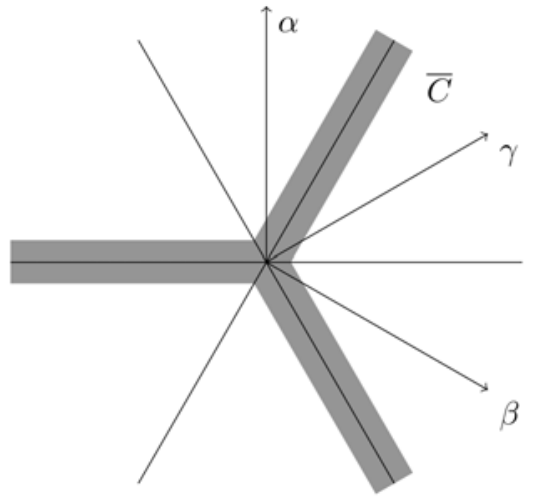


It follows that

$$
\begin{aligned}
L_{t}^{\beta}=\int_{0}^{t} \mathbf{1}_{\left.\left\{e^{i \pi / 3} \cdot X_{s}\right\rangle \geq 0\right\}} d L_{s}(\beta . X) & +\int_{0}^{t} \mathbf{1}_{\left\{e^{i \pi} \cdot X_{s} \geq 0\right\}} d L_{s}(\alpha . X) \\
& +\int_{0}^{t} \mathbf{1}_{\left\{e^{i 5 \pi / 3} \cdot X_{s} \geq 0\right\}} d L_{s}(\gamma \cdot X) .
\end{aligned}
$$

Acknowledgments. We thank J. P. Anker and P. Bougerol for helpful information and discussions on reflection groups. The research of the first author was partially supported by Agence Nationale de la recherche grant ANR-09-BLAN-0084-01.

\section{REFERENCES}

[1] P. Biane - P. Bougerol - N. O'Connell, Littelman paths and Brownian paths. Duke Math. J., 130 (2004), pp. 127-167.

[2] N. Bourbaki, Éléments de mathématiques: Groupes et algèbres de Lie. Chapitres 4-6, Hermann, Paris 1968.

[3] O. Chybiryakov, Processus de Dunkl et relation de Lamperti. Ph. D. Thesis, Université de Paris VI, 2006.

[4] O. Chybiryakov - L. Gallardo - M. Yor, Dunkl processes and their radial parts relative to a root system. Travaux en cours, 71 (Hermann 2008), pp. 113197.

[5] N. Demni, A guided tour in the world of radial Dunkl processes. Travaux en cours, 71 (Hermann 2008), pp. 199-226.

[6] J. E. Humpreys, Reflection Groups and Coxeter Groups. Cambridge University Press, 1990.

[7] D. REvUz - M. Yor, Continuous Martingales and Brownian Motion. Springer, third edition, 1999.

Manoscritto pervenuto in redazione il 5 gennaio 2011. 